\title{
Numerical solutions of 2-D incompressible driven cavity flow with wavy bottom surface
}

\author{
K. M. Salah Uddin ${ }^{1}$, Litan Kumar Saha ${ }^{2}$ \\ ${ }^{1}$ Department of Management Information Systems, University of Dhaka, Dhaka, Bangladesh \\ ${ }^{2}$ Deparment of Applied Mathematics, University of Dhaka, Dhaka, Bangladesh
}

Email address:

salahuddin@du.ac.bd (K. M. S. Uddin), 1ksaha.math@gmail.com (L. K. Saha)

\section{To cite this article:}

K. M. Salah Uddin, Litan Kumar Saha. Numerical Solutions of 2-D Incompressible Driven Cavity Flow with Wavy Bottom Surface. American Journal of Applied Mathematics. Special Issue: Fluid Flow and Heat Transfer Inside a Closed Domain. Vol. 3, No. 1-1, 2015, pp. 30-42. doi: $10.11648 /$ j.ajam.s.2015030101.14

\begin{abstract}
In the present numerical study is devoted to investigate the lid-driven cavity flow with wavy bottom surface. The cavity upper wall is moving with a uniform velocity by unity and the other walls are no-slip. The physical problem is represented mathematically by a set of governing equations and the developed mathematical model is solved by employing Galerkin weighted residual method of finite element formulation. The wide ranges of governing parameters, i. e., the Reynolds number $(\mathrm{Re})$, and the number of undulations $(\lambda)$ on the flow structures are investigated in detail. The behavior of the force coefficient $C_{f}$ also has been examined. Streamline plots provide the details of fluid flow. The fluid contained inside a squared cavity is set into motion by the top wall which is sliding at constant velocity from left to right and the undulation which was induced at the bottom surface. It is found that these parameters have significant effect on the flow fields in the cavity. Furthermore, the trends of skin friction for different values of the aforementioned parameters are presented in this investigation.
\end{abstract}

Keywords: Skin Friction; Lid Driven Cavity; Numerical Study, Wavy Surface

\section{Introduction}

Lid-driven flow in a two-dimensional square cavity is a classical, benchmark problem, which involves complex flow phenomena such as different types and scales of vortices, bifurcation, transition and turbulence. These important flow characteristics are closely relevant to a number of practical applications [1, 2]. Driven cavity flow serve as a benchmark problem for numerical methods in terms of accuracy, numerical efficiency and etc. Many studies can be found on the two-dimensional square cavity flow with its upper lid sliding at a constant speed. In fact, the literature review shows that two categories of studies were investigated [3]. The first category is concerned with a horizontal sliding lid, which encompasses the top wall [4-8], bottom-sliding wall [9], or an oscillating lid [10-12]. The second category is related with side driven differentially heated enclosures, where one wall or both vertical walls move with a constant speed [13-15].

Due to the simplicity of the cavity geometry and regular boundary conditions, applying a method on this flow considerably easiness and simplification in the numerical coding which allow to learn much about the fundamental issues underlying the steady flows in a finite closed enclosure Despite its simple geometry, the driven cavity flow retains a rich fluid flow physics manifested by multiple counter rotating recirculating regions on the corners of the cavity depending on the Reynolds number. Erturk et al [16] states that a new quaternary vortex at the bottom left corner and a new tertiary vortex at the top left corner of the cavity are observed in the flow field as the Reynolds number increases. Erturk and Gokcol [17] have investigated the 2-D steady incompressible flow inside a triangular cavity. Their study shows that the flow structures in a triangle cavity greatly affected by the triangular geometry. Migeon et al. [18] studied experimentally the effects of lid-driven cavity shape on the flow establishment phase for square, rectangular and semi-circular cavities.

Recently, Glowinski et al. [19] applied a finite element method to the wall-driven flow in a semi-circular cavity and revealed the vortex structure at high Reynolds numbers. Mercan and Atalik [20] have studied the high-Reynolds number lid-driven flow in arc-shape cavities with different cross sections is considered up to $\operatorname{Re}=8000$. They have 
discussed the effects of aspect ratio or arc angle, $r$, on the formation and growth of vertical structures, as well as on the existence and development of periodic solutions. The works of Ostrach [21], Catton [22] and Yang [23] which described the importance of the inclined cavity. Sheikholeslami et al. [24] studied the problem of natural convection between a circular enclosure and a sinusoidal cylinder. They concluded that streamlines, isotherms, and the number, size and formation of the cells inside the enclosure strongly depend on the Rayleigh number, values of amplitude and the number of undulations of the enclosure.

Most of the studies in the literature are concerned with the square or rectangular cavity flows, although in applications, the cavities may be non-rectangular. There are few studies dealing with flows in curved cavities driven by a moving lid. A sinusoidal wall is chosen for the bottom surface with different undulations. Therefore, in the present study, a numerical simulation has been conducted to realize the influence of the wavy bottom surface on the flow structures and skin friction in the square cavity.

\section{Mathematical formulation}

A two dimensional lid driven cavity of height and length L with wavy bottom surface is considered, as shown in Fig. 1. The shape of the bottom wavy surface profile is assumed to mock following the pattern $y=A(1-\cos (2 \lambda \pi x))$, where $\mathrm{A}$ is the dimensionless amplitudeof the wavy surface and $\lambda$ is the number of undulations. The upper wall of the cavity is allowed to move in its own plane at a constant speed unity and the other walls have no-slip condition. In general, the cavity fluid is assumed to be Newtonian and incompressible, unsteady and laminar flow. It is further assumed that viscous dissipation is neglected in this study. With abovementioned assumptions, the governing equations for conservations of mass and momentum can be written as



Fig. 1. Schematic diagram of the cavity and boundary conditions
Mass conservation equation:

$$
\frac{\partial u}{\partial x}+\frac{\partial v}{\partial y}=0
$$

Momentum conservation equation:

$$
\begin{aligned}
& u \frac{\partial u}{\partial x}+v \frac{\partial u}{\partial y}=-\frac{1}{\rho} \frac{\partial p}{\partial x}+v\left(\frac{\partial^{2} u}{\partial x^{2}}+\frac{\partial^{2} u}{\partial y^{2}}\right) \\
& u \frac{\partial v}{\partial x}+v \frac{\partial v}{\partial y}=-\frac{1}{\rho} \frac{\partial p}{\partial y}+v\left(\frac{\partial^{2} v}{\partial x^{2}}+\frac{\partial^{2} v}{\partial y^{2}}\right)
\end{aligned}
$$

where $\rho$ is the density of fluid, $\underline{u}=(u(x, y), v(x, y))$ the flow velocity vector, $p(x, y)$ the pressure, $\underline{x}=(x, y)$ the Cartesian coordinates and $v$ the kinematic viscosity of the fluid.

The governing equations (1)-(3) are non-dimensionalized using the following dimensional variables:

$$
X=\frac{x}{L}, Y=\frac{y}{L}, U=\frac{u}{U_{0}}, V=\frac{v}{U_{0}}, P=\frac{p}{\rho U_{0}^{2}}
$$

Introducing the above non-dimensional scales into the governing equations, we obtain the non-dimensional form of the equations as follows:

$$
\begin{gathered}
\frac{\partial U}{\partial X}+\frac{\partial V}{\partial Y}=0 \\
U \frac{\partial U}{\partial X}+V \frac{\partial U}{\partial Y}=-\frac{\partial P}{\partial X}+\frac{1}{\operatorname{Re}}\left(\frac{\partial^{2} U}{\partial X^{2}}+\frac{\partial^{2} U}{\partial Y^{2}}\right) \\
U \frac{\partial V}{\partial X}+V \frac{\partial V}{\partial Y}=-\frac{\partial P}{\partial Y}+\frac{1}{\operatorname{Re}}\left(\frac{\partial^{2} V}{\partial X^{2}}+\frac{\partial^{2} V}{\partial Y^{2}}\right) \\
U=1, V=0 \text { for } 0 \leq X \leq 1, Y=1 \\
U=V=0 \quad \text { for } 0 \leq X \leq 1, Y=A(1-\cos (2 \lambda \pi X)) \\
U=V=0 \quad \text { for } 0 \leq Y \leq 1, X=0, X=1
\end{gathered}
$$


as $\operatorname{Re}=\frac{U_{0} L}{v}$ where $U_{0}$ is the reference velocity, $v$ is the kinematic viscosity and $L$ is the reference scale velocity.

The relation defines skin friction at the upper wall $C_{f_{u}}=\frac{\mu \frac{\partial u}{\partial y} \mid y=1}{\frac{1}{2} \rho U_{0}^{2}}$

In non-dimensional form which becomes $C_{f_{u}}=\frac{2}{\operatorname{Re}} \frac{\partial U}{\partial Y} \mid Y=1$

Hence the total skin friction at the upper wall is $D=\int_{0}^{s} C_{f_{u}} d X$ 
The relation defines skin friction at the wavy lower wall

$$
C_{f_{l}}=\frac{\mu \frac{\partial u}{\partial y} \mid y=A(1-\operatorname{Cos}(2 \pi \lambda x))}{\frac{1}{2} \rho U_{0}{ }^{2}}
$$

In non-dimensional form which becomes $C_{f}=\frac{2}{\operatorname{Re}} \frac{\partial U}{\partial Y} \mid Y=A(1-\operatorname{Cos}(2 \pi \lambda X))$

Hence the total skin friction at the wavy lower wall $D=\int_{0}^{s} C_{f} d X$

\section{Numerical Method}

The governing equations along with the boundary conditions are solved through the Galerkin weighted residual finite element method. The formulation of this method and computational procedure is well described by Taylor and Hood [25] and Dechaumphai [26]. The computational domain is discretized employing the uniform mapped mesh grid system. Then the Galerkin weighted residual technique is used to convert the nonlinear governing partial differential equations into a system of integral equations that can be solved numerically. The integration involved in each term of these equations is performed by using Gauss's quadrature method, which leads to a set of non-linear algebraic equations. These equations are then modified by imposing boundary equations that is transferred into linear algebraic equations by Newton-Raphson iteration. Finally, these linear equations are solved by applying Triangular factorization method.

\section{Grid Refinement Study}

A numerical method is said to be convergent if the solution of the discretized equations tends to the exact solution as the grid spacing tends to zero. Convergence of a non-linear problem can be investigated by numerical tests, i.e. repeating the calculation on a series of successively refined grids. If the method is stable and all approximations used in the discretization process are consistent, we will find that the computation does converge to a grid-independent solution. A grid that was $50 \%$ coarser and finer than the base case grid was used to conduct the grid independency test. The computations at base case conditions were repeated on this refined grid and the solutions are compared. The numerical results and its accuracy greatly influenced by the size of the grid. In this study, we have adopted three types of grids, namely coarse grid, base grid and fine grid to examine the influence of grid resolution on the numerical results. The coarse grid and fine grid are the $50 \%$ refinement of the base case grid. The base case grid consists of $125 \times 125$ grid points where as the coarse grid consists of $71 \times 71$ grid points and the fine grid consists of $190 \times 190$ grid points. From Fig. 2 we observe that for coarse grid, velocity profile and skin friction profiles are deformed from the base line curve but the velocity profile for base grid and fine grid remain almost same. Therefore, we can consider base grid for computational procedure.
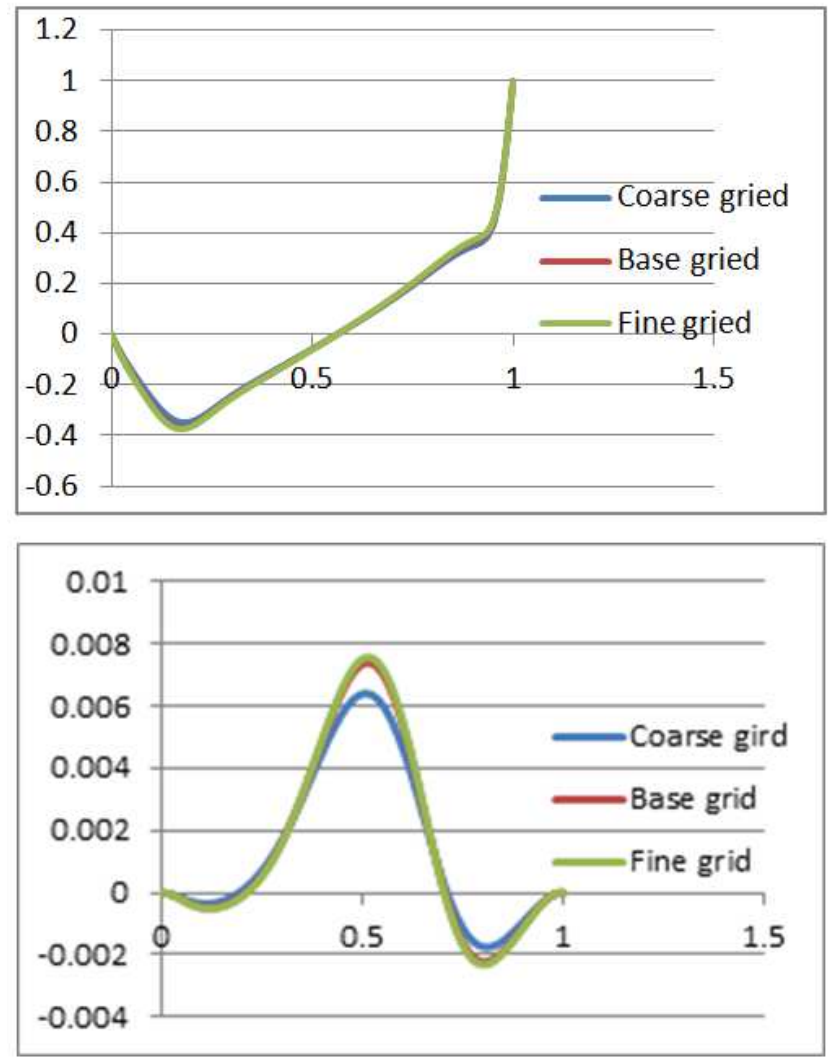

Fig. 2. (a) u-velocity profile along vertical line through the center of the cavity (b) variation of skin friction at the bottom wall for various grid point $, \lambda=0, R e=103$.

\section{Results and Discussions}

The characteristics of the flow fields in the lid-driven cavity are examined by exploring the effects of the Reynolds number $\mathrm{Re}$, number of undulations, and amplitude of the wavy bottom surface. Such field variables are examined by outlaying the steady state version of the streamlines. The model validation is an essential part of a numerical investigation. Hence, the outcome of the present numerical code was benchmarked against the numerical results of Poochinapan and Ching Mai [28], which were reported for two-dimensional incompressible flow in a lid-driven cavity. The comparison was conducted while employing the following dimensionless parameters: $\operatorname{Re}=100,5000$. Excellent agreement was achieved, as illustrated in Fig. 3(a) \& 3(c), between our results and the numerical results of Poochinapan and Ching Mai [28]] for the streamlines inside the cavity. Further validation was performed by comparing the streamlines contour in the lid-driven square cavity between the present work and that of Moshkin \& Poochinapan [27] while employing Reynolds number $\mathrm{Re}=1000$ depicted in Fig. 3(b). Again, the comparison strikes excellent agreement between both results as displayed in Fig. 3(b). These validations boost the confidence in our numerical code to carry on with the above stated objectives of the current investigation. 
$\operatorname{Re}=1 \times 10^{2}$

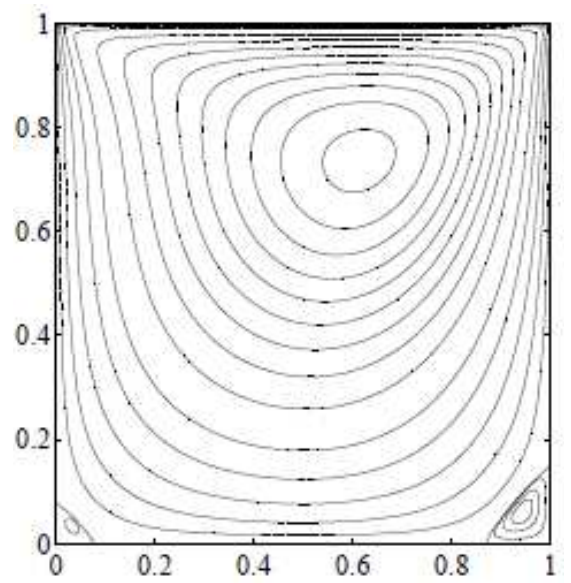

(a)

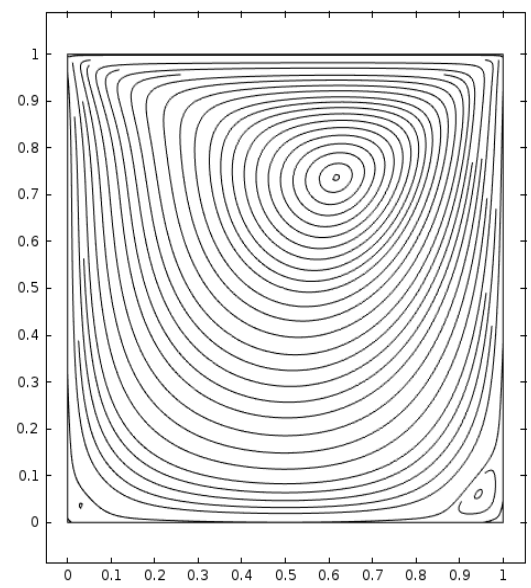

(d)
$\operatorname{Re}=1 \times 10^{3}$



(b)

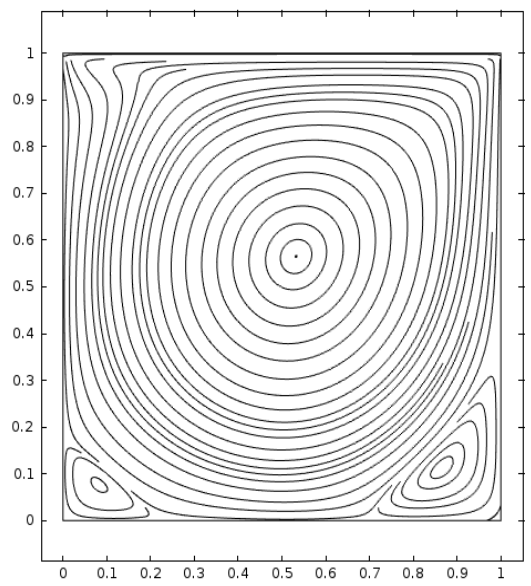

(e)
$\operatorname{Re}=5 \times 10^{3}$



(c)

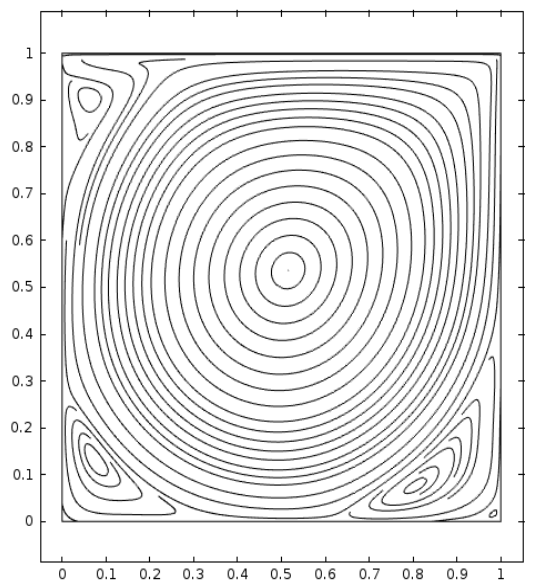

(f)

Fig. 3. Streamline contour with a comparison to Poochinapan and Mai[28]( (a)\& (c)) Moshkin and Poochinapan [27](b), present result (d), (e) \& (f).

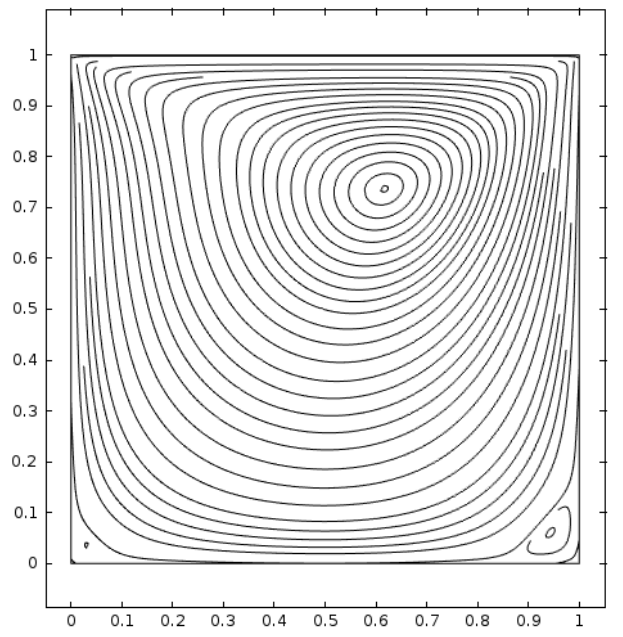

(a)



(b) 


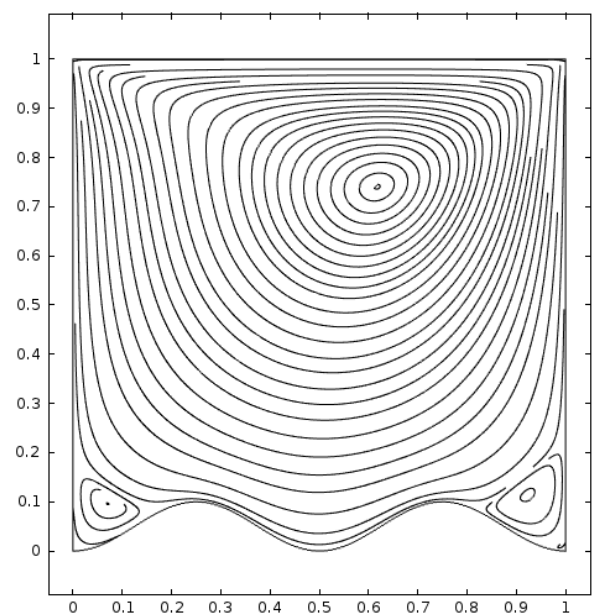

(c)

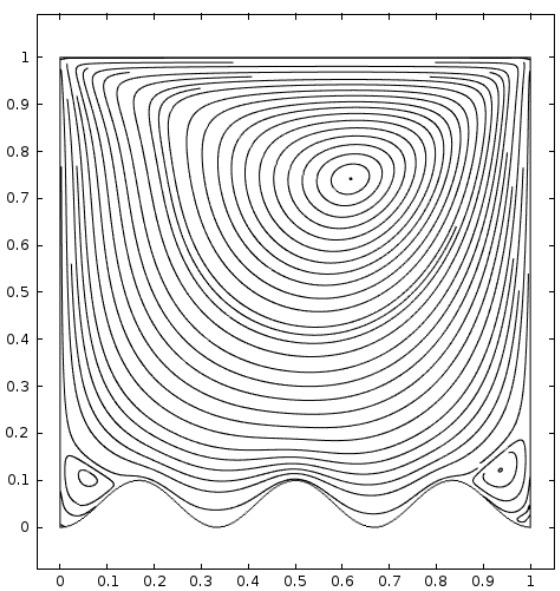

(d)

Fig. 4. Streamline contour at $\mathrm{Re}=1 \times 10^{2}, A=0.05$ for (a) $\lambda=0 \quad$ (b) $\lambda=1$ (c) $\lambda=2$ (d) $\lambda=3$

The effect of number of undulation on the streamline contour is depicted in Fig. 4 at $\mathrm{Re}=1 \times 10^{3}$ from which we observed that undulation of the bottom surface affect the eddy on the bottom right corner and bottom left corner. A small eddy appears at right lower corner when undulation is not employed at the bottom lower surface. However, employing undulation at the bottom lower surface, a very small eddy occurs on the left and right bottom corner. Also in Fig. 4(d), we see that very small tertiary eddy occur in the left and right bottom corner, which indicate that the increasing the number of undulation affect the flow to the turbulent direction.

The streamlines contour are illustrated in Fig. 5 for various undulations at Reynolds number, $\mathrm{Re}=1000$. from which we observed that for zero, first and second undulation large recirculation in the clockwise direction occur in the bottom right and bottom left corner also for second undulation tertiary eddy occur in the bottom right corner. We see from Fig. 5(d) for third undulation on the concave up region secondary eddy formulate and a small tertiary eddy occur on the bottom right and bottom left corner. The influence of number of undulations on the streamline contour are demonstrated in Fig. 7 at $R e=5000$. It is observed from the figure that for first undulation secondary and tertiary eddy occurs in the bottom right and bottom left corner. From Fig. 6(c), we see that secondary and tertiary eddy occur in the bottom left and bottom right corner and a secondary eddy occur in the concave up region. From Fig. 6(d) we see that due to the symmetric property of flow in two dimensional recirculating flow in the clock wise direction that occur in the concave up region co-operate with the recirculating flow that occur in the bottom right and bottom left corner.

(a)

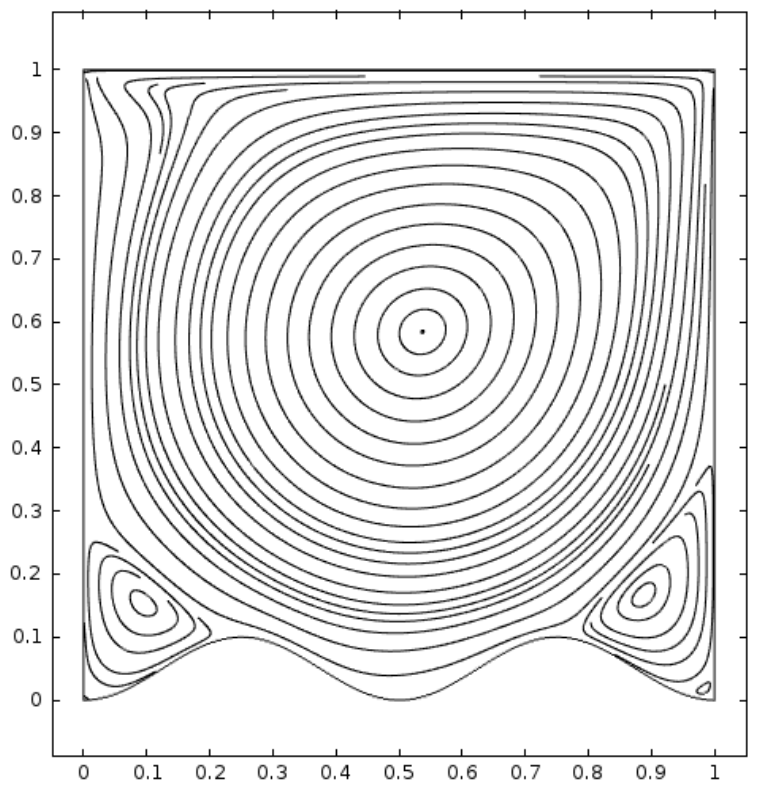

(c) (b)

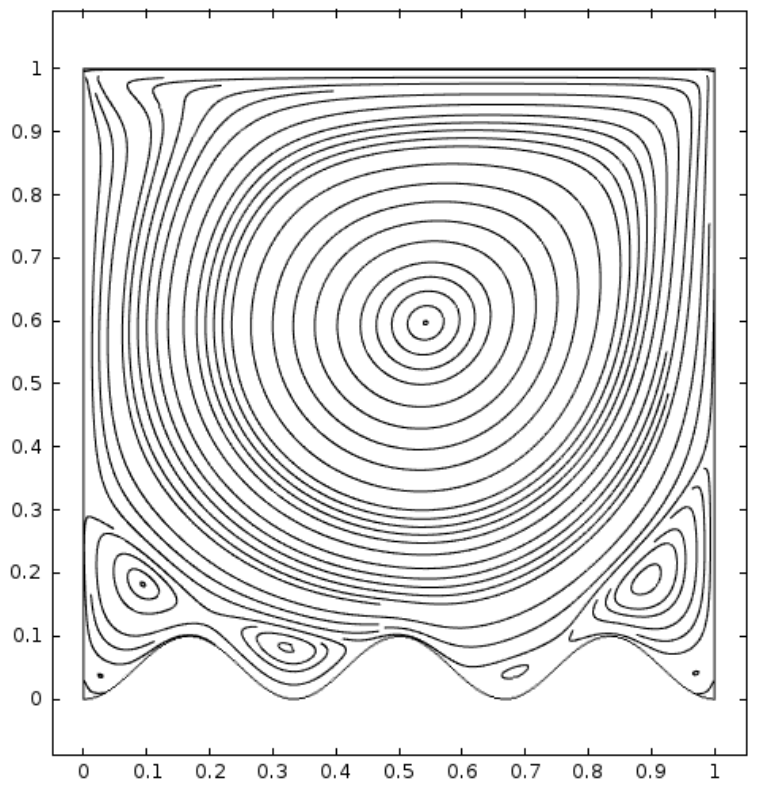

(d)

Fig. 5. Streamline contour at $\operatorname{Re}=1 \times 10^{3}, A=0.05$ for (a) $\lambda=0$ (b) $\lambda=1$ (c) $\lambda=2$ (d) $\lambda=3$

The vortices contour for the amplitude of the wavy bottom surface $A=0.05$ and various number of undulations $\lambda=0,1,2,3$ is shown in Fig. 7. It was observed from Fig. 7 that vortices clustered near the left and right corner of the upper wall also some vortices occur in the upper half of the cavity. Also for undulations, small vortices occur at the upper pick point of the wave. The vorticity contour for the amplitude of the wavy bottom surface $A=0.05$ and various number of undulations number $\lambda=0,1,2,3$ is shown in Fig. 8. From this figure, it was observed that vortiicity decreased in the bulk of the cavity and clustered near the boundary and the undulations of the bottom surface affect the vortices.

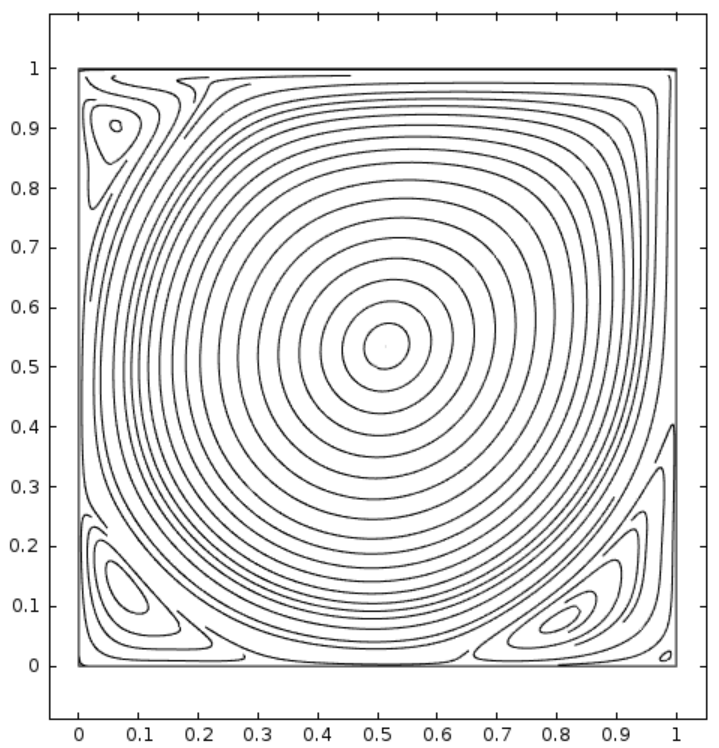

(a)



(b) 


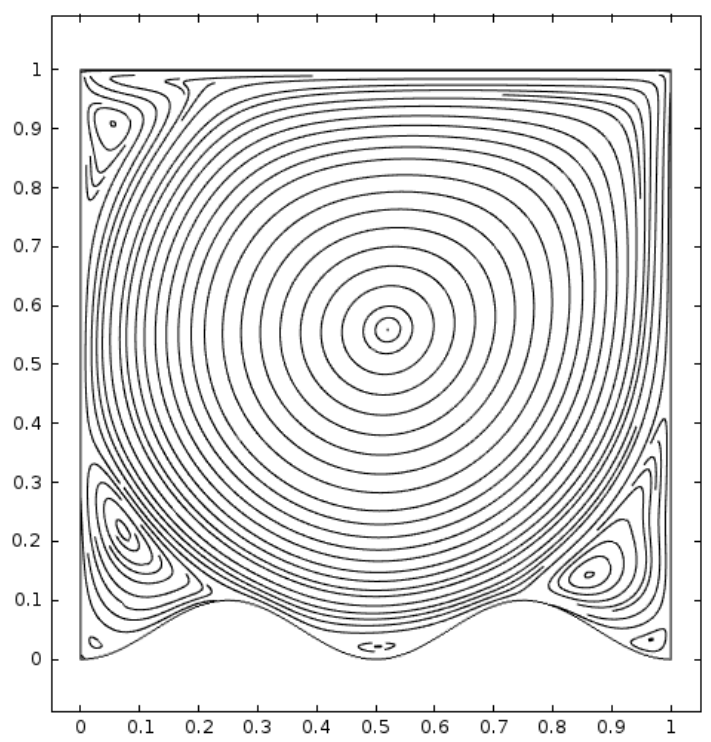

(c)

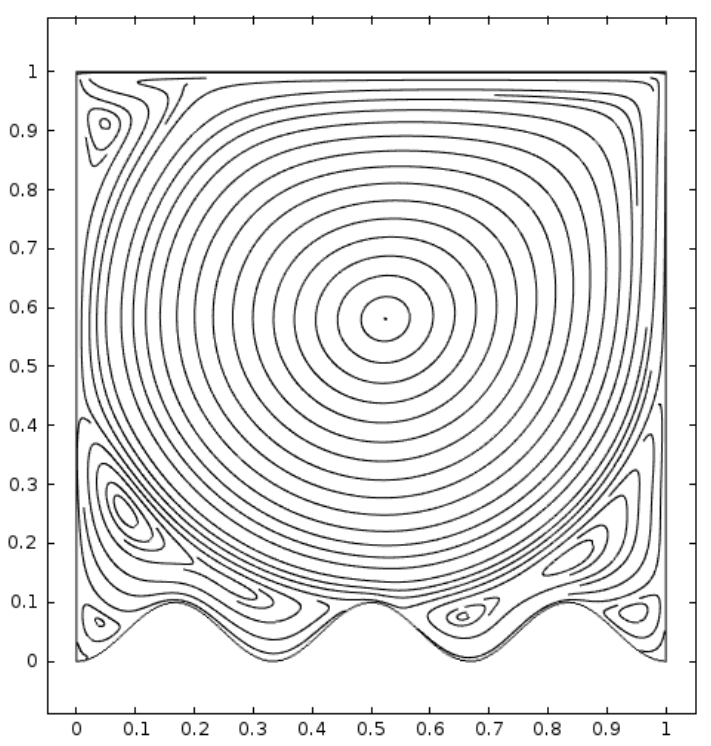

(d)

Fig. 6. Streamline contour at $\operatorname{Re}=5 \times 10^{3}, A=0.05$ for (a) $\lambda=0 \quad$ (b) $\lambda=1$ (c) $\lambda=2$ (d) $\lambda=3$

The vorticity contour for and for the amplitude of the wavy bottom surface $A=0.05$ (a) $\lambda=0$ (b) $\lambda=1$ (c) $\lambda=2$ (d) $\lambda=3$ is shown in Fig. 9. From which it was observed that the vortices more clustered near the boundary and there is no vortices occur in the bulk of the cavity. These figures show the vortices that are formed in the flow field increases as the Reynolds number increases. The v-component velocity profile for various undulations along horizontal line through the center of the cavity has been presented in Fig. 10. It is observed that for low value of the Reynolds number, v-velocity along horizontal line through the cavity slightly affect near the left and right wall but for higher value of the Reynolds number effect of undulations on the v-velocity profile decreases. The $\mathrm{u}$-component velocity profile for various undulations along vertical line through the center of the cavity is depicted in Fig. 11. From which it is observed that for low value of the Reynolds number there is not accountable effect on the u-velocity but for higher value of the Reynolds number u-velocity profile affected by undulations of the bottom surface near the bottom surface and effect of undulation decreases as the value of $y$ increases.

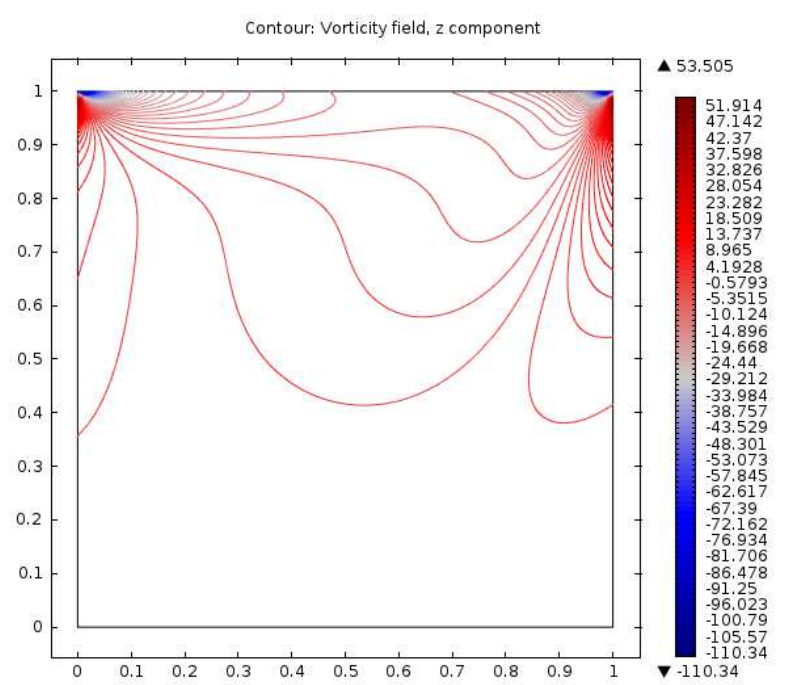

(a)



(b) 


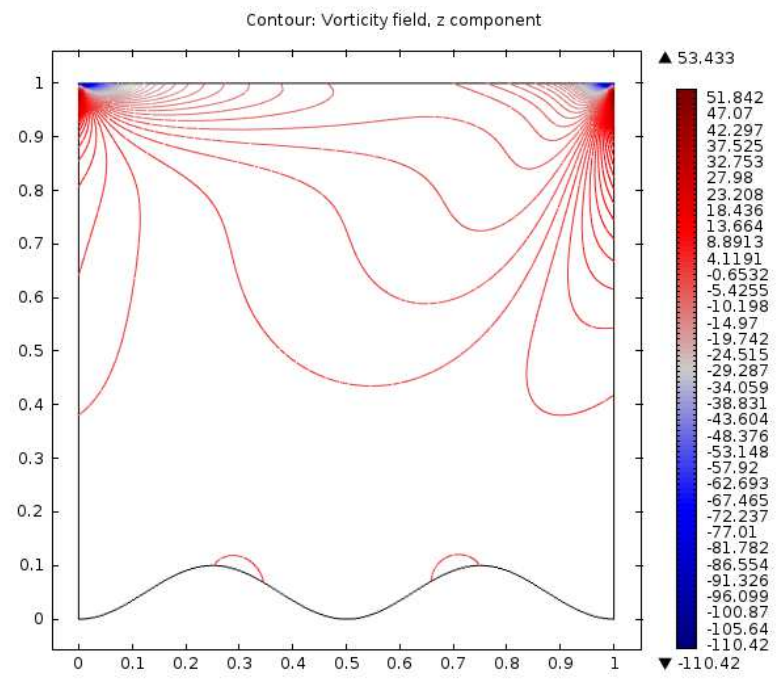

(c)



(d)

Fig. 7. Vorticity contour for $\mathrm{Re}=1 \times 10^{2}, A=0.05$ (a) $\lambda=0$ (b) $\lambda=1$ (c) $\lambda=2$ (d) $\lambda=3$

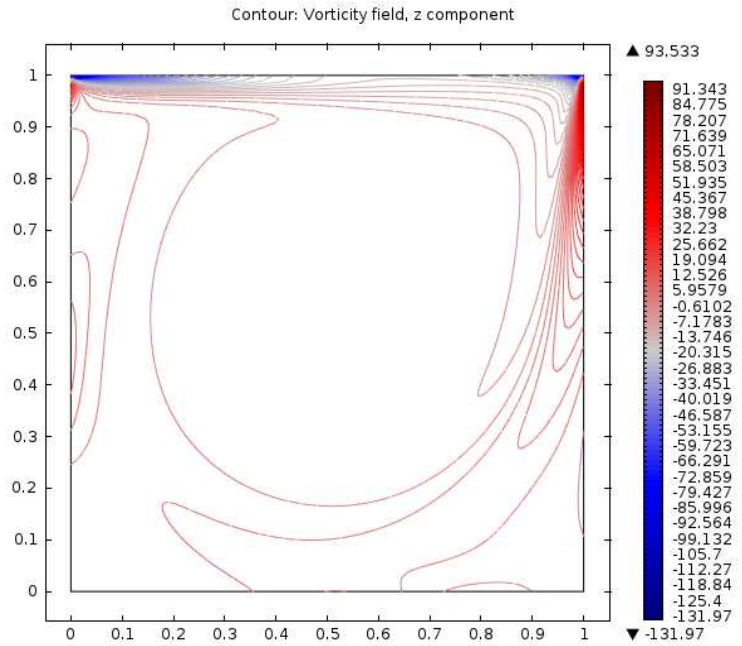

(a)

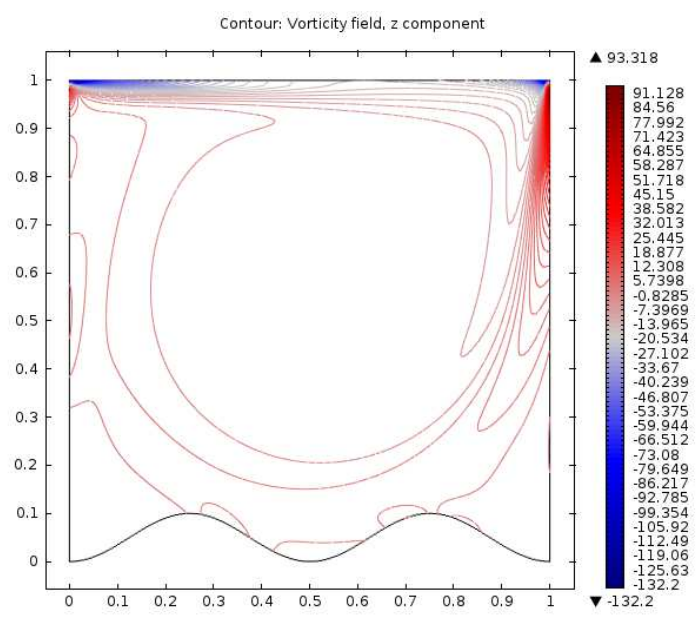

(c)

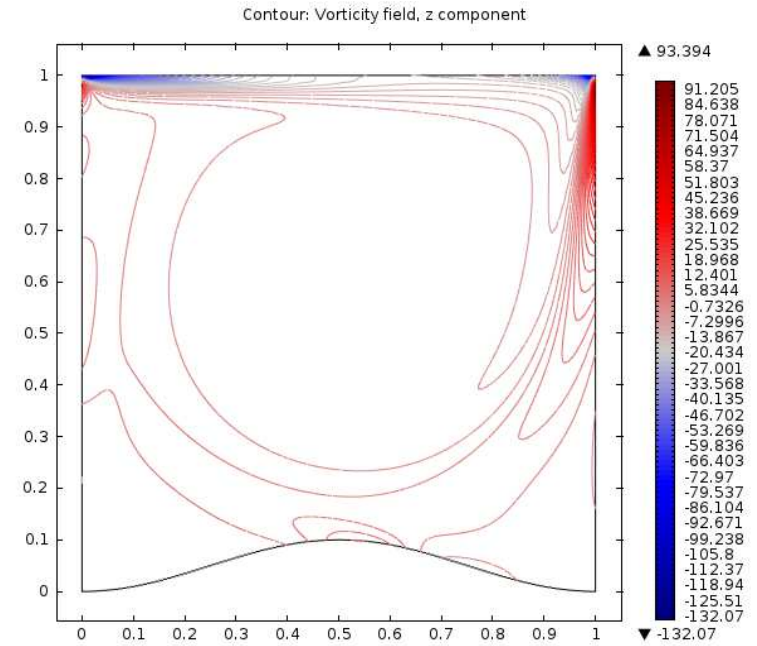

(b)



(d)

Fig. 8. Vorticity contour for $\operatorname{Re}=1 \times 10^{3}, A=0.05$ (a) $\lambda=0$ (b) $\lambda=1$ (c) $\lambda=2$ (d) $\lambda=3$ 
Contour: Vorticity field, $z$ component

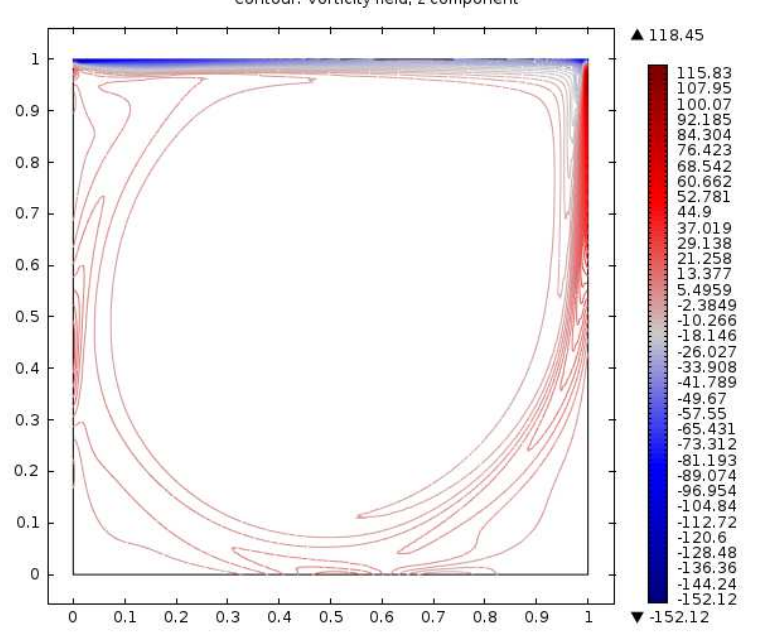

(a)

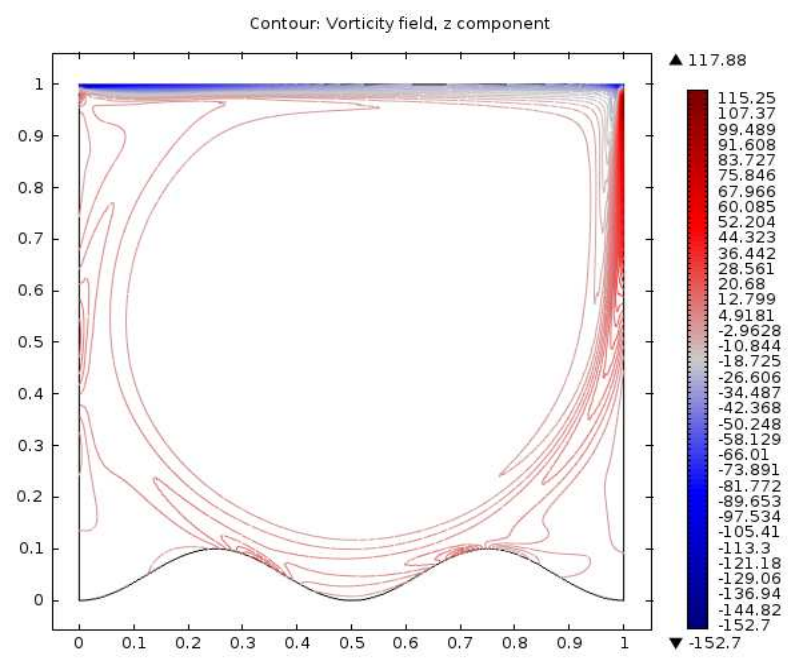

(c)

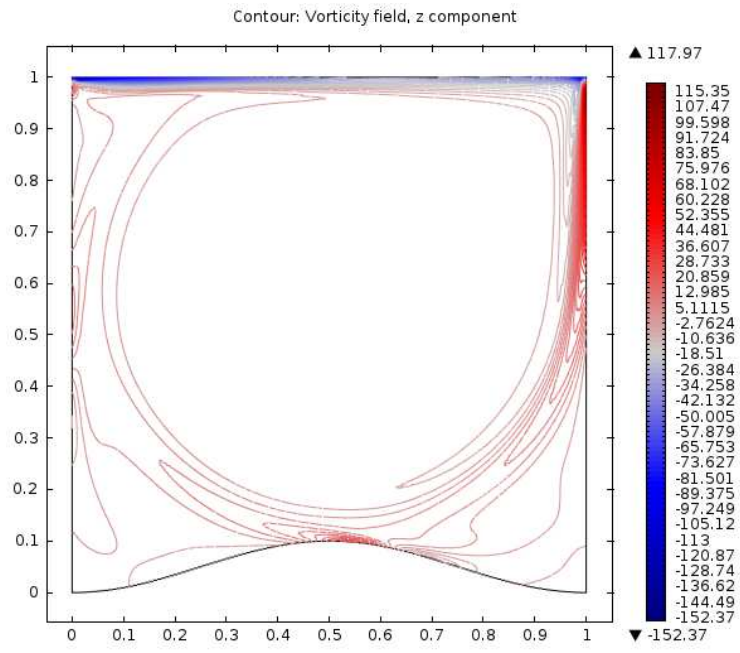

(b)



(d)

Fig. 9. Vorticity contour for $\operatorname{Re}=5 \times 10^{3}, A=0.05$ (a) $\lambda=0$ (b) $\lambda=1$ (c) $\lambda=2$ (d) $\lambda=3$

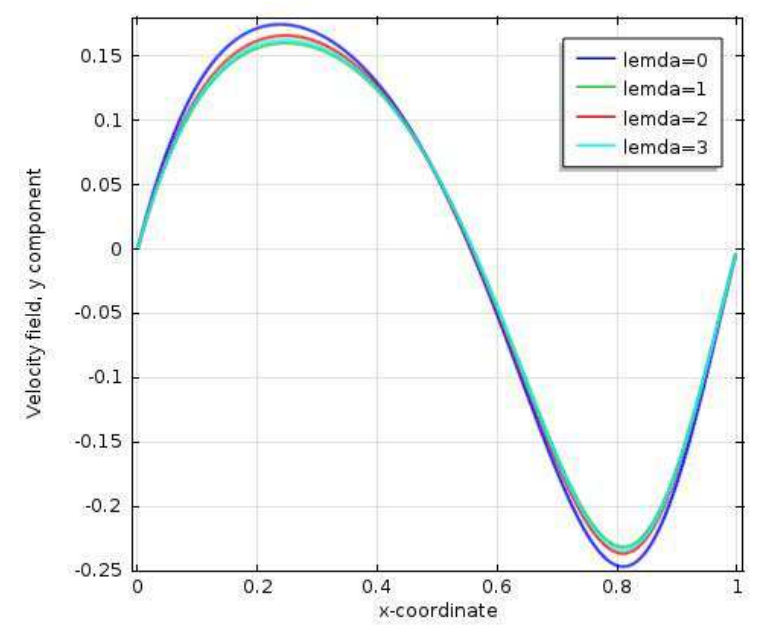

(a)



(b) 




(c)

Fig. 10. $v$-velocity profile for various undulations along horizontal line through the center of the cavity, $A=0.05$ (a) $\operatorname{Re}=1 \times 10^{2}\left(\right.$ b) $\operatorname{Re}=1 \times 10^{3}$ (c) $\mathrm{Re}=5 \times 10^{3}$.

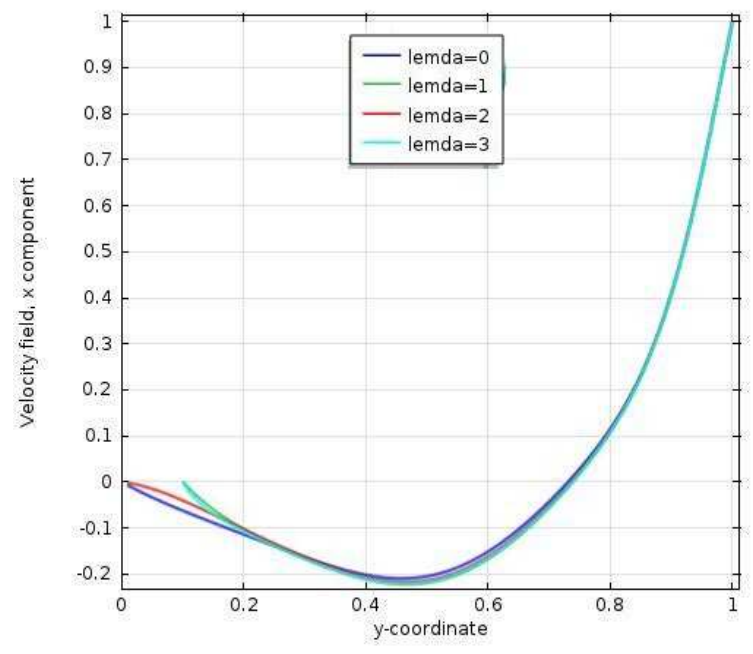

(a)



(b)

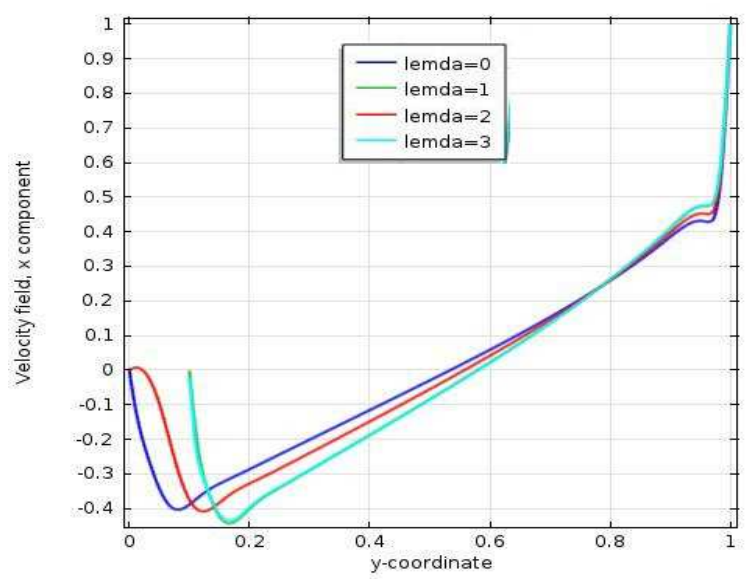

(c)

Fig. 11. $u$-velocity profile for various undulations along vertical line through the center of the cavity, $A=0.05$ (a) $\operatorname{Re}=1 \times 10^{2} ;$ (b) $\operatorname{Re}=1 \times 10^{3} ;$ (c) $\operatorname{Re}=5 \times 10^{3}$. 


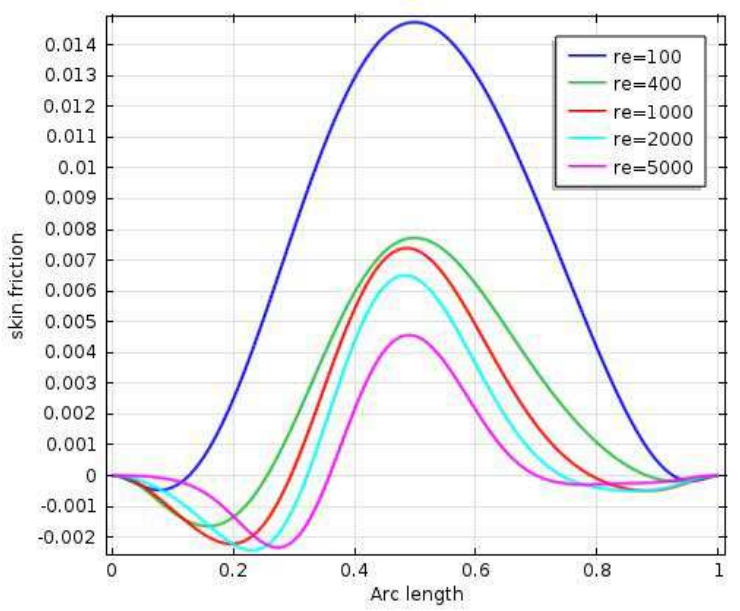

(a)



(c)



(b)

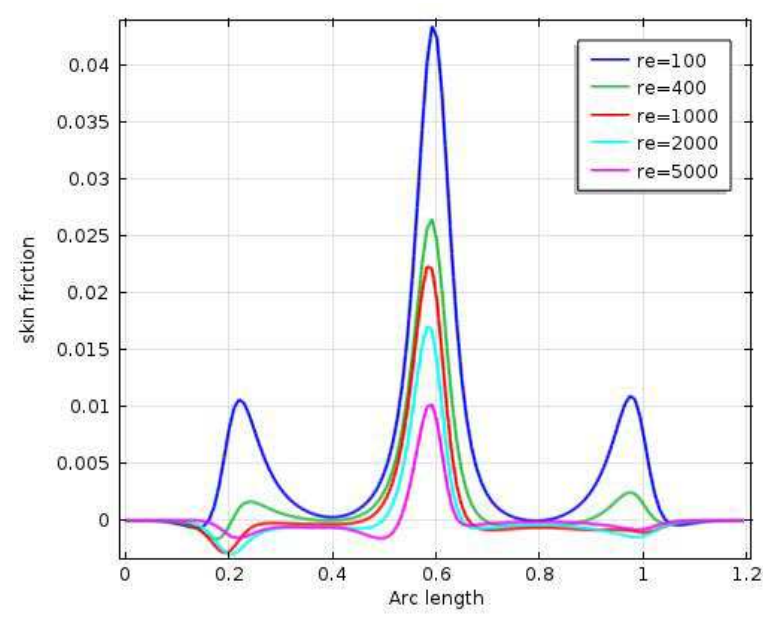

(d)

Fig. 12. Variation of skin friction along bottom surface (a) $\lambda=0$ (b) $\lambda=1 \quad$ (c) $\lambda=2$ (d) $\lambda=3$

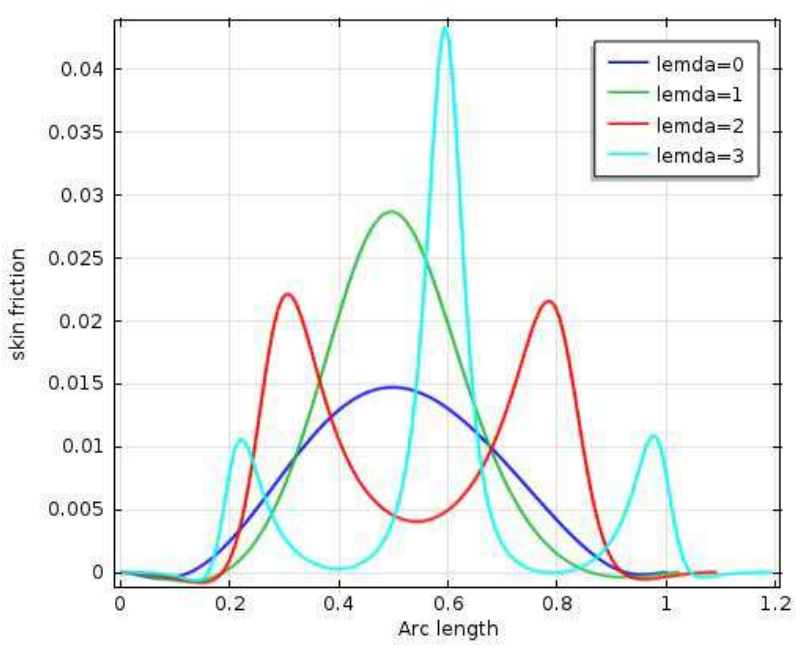

(a)

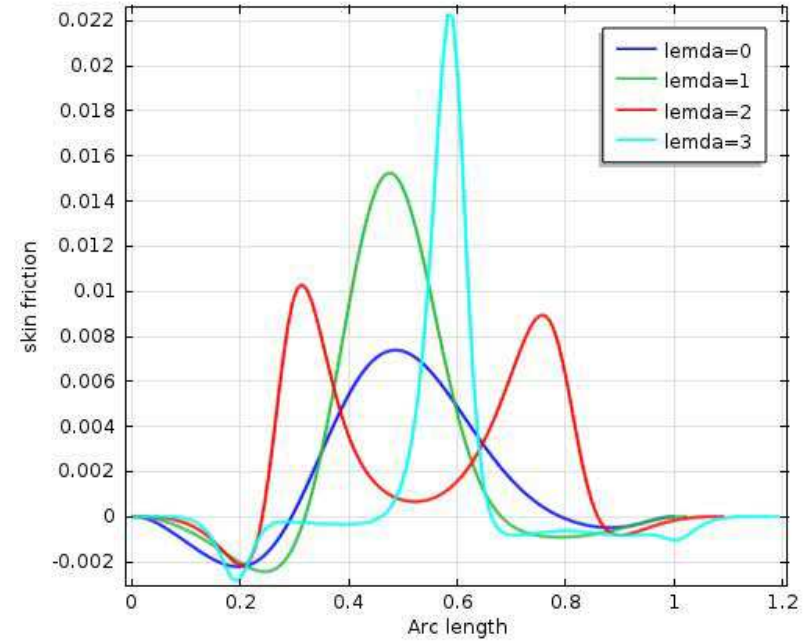

(b) 


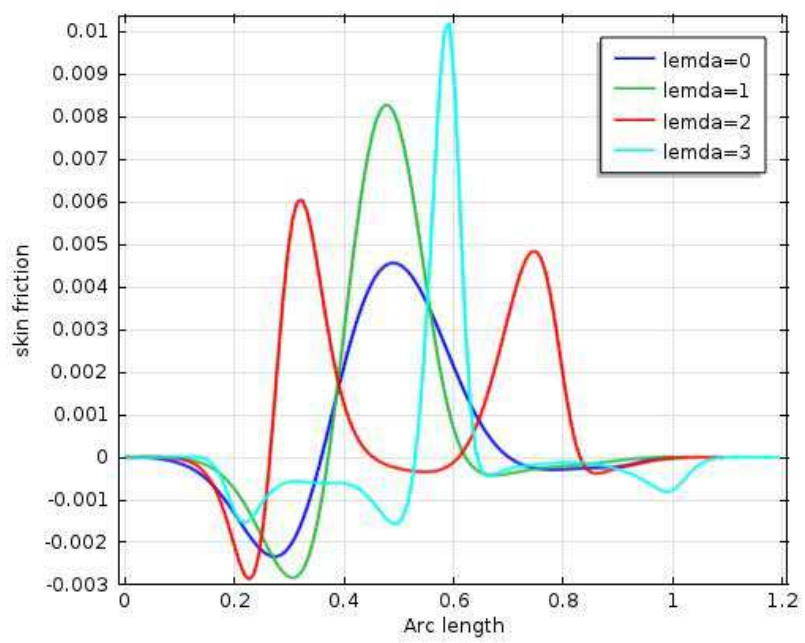

(c)

Fig. 13. Skin friction along wavy bottom surface for various undulation (a) $\operatorname{Re}=1 \times 10^{2}$ (b) $\operatorname{Re}=1 \times 10^{3}$ (c) $\operatorname{Re}=5 \times 10^{3}$

Fig. 12 represents the variation of skin friction for Reynolds number, $R e=1 \times 10^{2}, 1 \times 10^{3}, 5 \times 10^{3}$. From Fig. 12(a) we observed that negative skin friction for $\lambda=0$ become maximum near at the middle point of the cavity lower boundary and it became minimum between the points $x=0.2$ and $x=0.3$. For $\lambda=1$ negative skin friction profile look like similar as for $\lambda=0$ but the values of negative skin friction grow for undulation at the pick point. From Fig. 12(c) and Fig. 12(d) we see that the negative skin friction rise depending on the increasing of the curve and it became maximum at maximum point but it decrease as the curve decrease become minimum at local minimum point but for higher Re have some complicated nature near the left and right boundary wall.

Fig. 13 represents the negative skin friction for various values of Re. From Fig. 13(a) we observed that for skin friction smoothly increases for $\lambda=0$ and it becomes maximum the middle point of the lower surface and then decreases to zero at the right boundary. But employing the number of undulations at bottom surface the skin friction increases as the surface increases. The highest skin friction is found at the upper peak point. It decreases again when the curve goes downward. Thereafter, the lowest skin friction is obtained at the lower peak point. From Fig. 13(b) we observed that for flat surface, the skin friction slightly decreases near the left boundary and then increasing but the nature of the skin friction remain same as for except the maximum value of skin friction become lower. From Fig.13(c) we observed that it has the same nature as for except the maximum and minimum value.

\section{Conclusion}

In the present work deals with the effects of undulated bottom surface on a lid driven square cavity flow. In this paper we conducted a simulation of incompressible viscous flow in a lid-driven square cavity, described the cavity behavior of this flow at the high Reynolds numbers, examined effects of Re, number of undulations and the grid size on numerical solutions of streamlines. The results obtained show that the wall undulation affects the flow in the lid driven square cavity. Employing the number of undulations at bottom surface, the skin friction increases. The skin friction becomes highest at the upper peak point and it becomes lowest at the lower peak point.

\section{References}

[1] Shankar PN, Deshpande MD., Fluid mechanics in the driven cavity, Ann Rev Fluid Mech 2000; 32:93-136.

[2] Robert NM, Pavageau M, Rafailidis S, Schatzmann M. Study of line source characteristics for 2-D physical modeling ofpollutant dispersion in street canyons, Journal of Wind Engineering and Industrial Aerodynamics, 1996; 62: 37-56.

[3] R. Iwatsu, J.M. Hyun, Three-dimensional driven-cavity flows with a vertical temperature gradient, Int. J. Heat Mass Transfer 38 (1995)3319-3328.

[4] A.A. Mohamad, R. Viskanta, Transient low Prandtl number fluid convection in a lid-driven cavity, Num. Heat Transfer: Part A 19(1991) 187-205.

[5] A.K. Prasad, J.R. Koseff, Combined forced and natural convection heat transfer in a deep lid-driven cavity flow, Int. J. Heat Fluid Flow 17 (1996) 460-467.

[6] C.F. Freitas, R.L. Street, Non-linear transient phenomena in a complex recirculating flow: A numerical investigation, Int. J. Num. Methods Fluids 8 (1988) 769-802

[7] A.A. Mohamad, R. Viskanta, Flow and heat transfer in a lid-driven cavity with stably stratified fluid, Appl. Math. Model 19 (1995) 465-472.

[8] K.M. Khanafer, A.J. Chamkha, Mixed convection flow in a lid driven enclosure with a fluid-saturated porous medium, Int. J. Heat Mass Transfer 42 (1999) 2465-2481.

[9] C.-J. Chen, H. Nassari-Neshat, K.-S. Ho, Finite-analytical numerical solution of heat transfer in two-dimensional cavity flow, Num. Heat Transfer 4 (1981) 179-197. 
[10] R. Iwatsu, J.M. Hyun, K. Kuwahara, Convection in a differentially heated square cavity with a torsionally-oscillating lid, Int. J. Heat Mass Transfer 35 (1992) 1069-1076.

[11] R. Iwatsu, J.M. Hyun, K. Kuwahara, Numerical simulation of flows driven by a torsionally oscillating lid in a square cavity, J. Fluids Eng. 114 (1992) 143-149.

[12] Abdalla Al-Amiri, K. Khanafer, I. Pop, Numerical simulation of unsteady mixed convection in a driven cavity using an externally excited sliding lid, Eur. J. Mech. B/Fluids, in press

[13] V.S. Arpaci, P.S. Larsen, Convection Heat Transfer, Prentice-Hall, 1984, p. 90.

[14] O. Aydın, Aiding and opposing mechanisms of mixed convection in a shear-and buoyancy-driven cavity, Int. Comm. Heat Mass Transfer 26 (1999) 1019-1028.

[15] A.J. Chamkha, Hydromagnetic combined convection flow in a vertical lid-driven cavity with internal heat generation or absorption, Num. Heat Transfer: Part A 41 (2002) 529-546.

[16] E. Erturk, T. C. Corke2 and C. C. Gokcol, Numerical solutions of 2-D steady incompressible driven cavity flow at high Reynolds numbers, International Journal For Numerical Methods In Fluids, 2005; 48:747-774

[17] E. Erturk and O. Gokcol, Fine grid numerical solutions of triangular cavity flow, The European Physical Journal Applied Physics, 2007, 38, 97-105

[18] C. Migeon, A. Texier, G. Pineau, Effects of lid driven cavity shape on the flow establishment phase, J. Fluids Struct. 14 (2000) 469-488.

[19] R. Glowinski, G. Guidoboni, T.W. Pan, Wall driven incompressible viscous flow in a two-dimensional semi-circular cavity, J. Comput. Phys. 216 (2006) 76-91.
[20] H. Mercan, K. Atalık, Vortex formation in lid-driven arc-shape cavity flows at high Reynolds numbers, European Journal of Mechanics B/Fluids 28 (2009) 61-71

[21] S. Ostrach, Natural convection in enclosures, in: J.P. Hartnett, T.F. Irvine Jr. (Eds.), Advances in Heat Transfer, vol. 8, Academic Press, New York, 1972, pp. 161-227.

[22] I. Catton, Natural convection in enclosures, in: Proceedings of the Sixth International Heat Transfer Conference, vol. 6, 1978, pp. 13-31.

[23] K.T. Yang, Transitions and bifurcations in laminar buoyant flows in confined enclosures, J. Heat Transfer 110 (1988) 1191-1204.

[24] Sheikholeslami, M., Gorji-Bandpy, M., Pop, I. \& Soleimani, S. (2013). Numerical study of natural convection between a circular enclosure and a sinusoidal cylinder using control volume based finite element method. International Journal of Thermal Sciences, Vol. 72, pp. 147-158.

[25] C. Taylor, P. Hood, A numerical solution of the Navier-Stokes equations using finite element technique, Computers \& Fluids 1 (1) (1973) 73-89

[26] P. Dechaumphai, Finite Element Method in Engineering, 2nd ed. Chulalongkorn University Press, Bangkok, 1999.

[27] N. P. Moshkin, K. Poochinapan, Novel finite difference scheme for the numerical solution of two-dimensional incompressible Navier-Stokes equations, International Journal Of Numerical Analysis And Modeling, 7(2)(2010) 321-329

[28] K. Poochinapan and Chiang Mai, Numerical Implementations for 2-D Lid Driven Cavity Flow in Stream Function Formulation”, ISRN Applied Mathematics, 2012 (2012), pp. $1-17$. 